\title{
When ChIA PETs meet Hi-C
}

Two methods enable the drawing of genome-wide chromatin interaction maps: one looks at protein-independent folding principles, the other at protein-mediated functional interactions.

In its stretched out form, human chromatin adds up to a roughly two meter long polymer that needs to fit into a nucleus approximately ten micrometers in diameter. In addition, genes and their regulatory elements need to communicate, and chromatin must open to allow transcription and replication. Important questions are 'how does the linear order of genes relate to their spatial arrangement?' and 'how are genes regulated by distant elements?'. Two independent groups recently tackled these questions from opposite directions.

Job Dekker from the University of Massachusetts and Eric Lander from the Broad Institute headed an effort to gain an unbiased view of chromatin organization in the nucleus. "We wanted to have a technique to look at the structure of an entire chromosome at kilobase resolution," recalls Dekker. Unlike previous chromatin conformation capture methods that interrogate the interaction between two loci (3C), between one locus and the rest of the genome (4C) or between multiple loci (5C), their Hi-C technique captures interactions genome-wide (Lieberman-Aiden et al., 2009). Hi-C is a $3 \mathrm{C}$ derivative, based on proximity ligation of cross-linked DNA with a biotin linker, which is used to pull down the fragments, followed by high-throughput sequencing. From eight million read pairs, the scientists generated a genome-wide contact matrix at one-megabase resolution.

Dekker remarks that the difficulty for $\mathrm{Hi}-\mathrm{C}$ is not so much in the chromatin capture but in the interpretation of the data. "There are pronounced polymer features in the data that determine the background," he says. "It is not homogenous." He speculates that to really see the three-dimensional structure of a chromosome at the desired

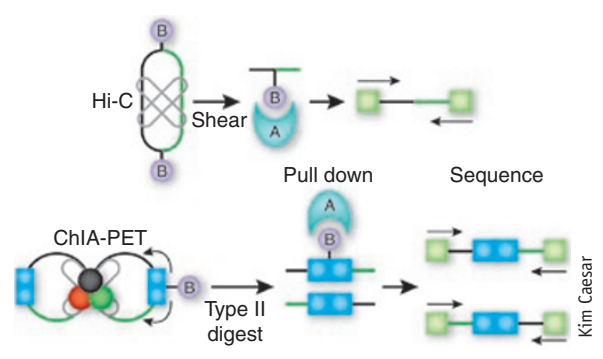

Schematic of Hi-C and ChIA-PETs.

one-kilobase resolution, hundreds of millions of reads will be needed in addition to a new way to model the interaction data.

But in the meantime, the scientists found surprising results with the number of reads they already have: chromatin does not resemble a compact, densely knotted structure but rather a fractal globule, a knot-free, tightly packed conformation in which active and inactive chromatin domains are folded together in two spatially different compartments.

Although this global arrangement allows insights into chromatin folding principles, at this point it is not clear whether spatial proximity of two loci necessarily means that there is a functional relationship; this, according to Dekker, will require more detailed analysis of these loci.

Yijun Ruan and Edwin Cheung from the Genome Institute of Singapore were interested in these functional relationships between a subset of loci. They asked specific questions, namely, 'how does a single transcription factor affect the chromatin interactome?' and 'why are so many transcription factor binding sites a long distance away from the gene promoters?'. For answers, they elegantly combined the existing methods of chromatin immunoprecipitation (ChIP) and paired-end ditag (PET) sequencing. The researchers ligated immunoprecipitated DNA fragments to a barcoded linker with a type II restriction enzyme that created the PETs; they then subjected these chromatin interaction (ChIA) PETs to high-throughput sequencing, which yielded the transcription factor-dependent interaction map at base-pair resolution (Fullwood et al., 2009).

For Ruan, the big issue in data interpretation was how to deal with the noise. "Noise comes from two levels," he says, "biological and technical." Biological noise is caused by the dynamic nature of chromatin: as it is constantly moving, many interactions at any given point in time will be meaningless. Ruan's theory is that meaningful interactions will be stronger and thus withstand harsher fractionation methods.

The technical noise originates in the proximity ligation step when DNA fragments from different complexes ligate. To control for this chimera formation, Ruan's team split the chromatin material into two aliquots, then added linkers with two different barcodes before mixing the aliquots again before ligation. Chimeras, ligation products from different complexes, will have different linkers in the same PET and can be excluded. The researchers also carefully controlled that the interactions they saw were indeed functional.

It is Ruan's goal to now generate a more generic map of all interactions involved in transcription. "With Hi-C you hope to capture all interactions," Ruan says, "but you do not have their function. With ChIA PETs, we can experimentally annotate each of the interaction sites."

Superimposing Hi-C maps and ChIAPET maps with existing annotation will allow understanding gene regulation in three dimensions.

\section{Nicole Rusk}

\section{RESEARCH PAPERS}

Fullwood, M.J. et al. An oestrogen-receptor- $\alpha$-bound human chromatin interactome. Nature 462, 58-64 (2009).

Lieberman-Aiden, E. et al. Comprehensive mapping of long-range interactions reveals folding principles of the human genome. Science 326, 289-293 (2009). 